July 31,2021

hep-th/0212235

MIT-CTP-3334

\title{
Comments on Condensates in Non-Supersymmetric Orbifold Field Theories
}

\author{
David Tong \\ Center for Theoretical Physics, Massachusetts Institute of Technology, \\ Cambridge, MA 02139, U.S.A. \\ dtong@mit.edu
}

\begin{abstract}
Non-supersymmetric orbifolds of $\mathcal{N}=1$ super Yang-Mills theories are conjectured to inherit properties from their supersymmetric parent. We examine this conjecture by compactifying the $Z_{2}$ orbifold theories on a spatial circle of radius $R$. We point out that when the orbifold theory lies in a specific weakly coupled vacuum, fractional instantons do give rise to the conjectured condensate of bi-fundamental fermions. However, we show that quantum effects render this vacuum unstable through the generation of twisted operators. In the true vacuum state, no fermion condensate forms. Thus, in contrast to super YangMills, the compactified orbifold theory undergoes a chiral phase transition as $R$ is varied.
\end{abstract}




\section{Introduction}

The holomorphic properties of supersymmetric gauge theories allow us to calculate certain quantities exactly, even in strongly coupled regimes. However, to make progress in more realistic, non-supersymmetric theories, we must learn to abandon our holomorphic comfort blanket.

The "orbifold field theory" is an interesting attempt in this direction which grew out of considerations in string theory. This method truncates a parent theory to a subset of fields which are left invariant under a discrete group action $G$. By suitably embedding $G$ in the parent R-symmetry group, non-supersymmetric daughter theories may be constructed from supersymmetric parents. Nevertheless, the daughter and parent theories enjoy the same planar graph expansion [1, 2, 3, 4]. It has been conjectured by Strassler that this large N correspondence continues to hold for non-perturbative effects [5]. More precisely, the conjecture states that if the parent and orbifold theories share a common vacuum then the Green's functions for their shared operators will coincide. The importance of this conjecture lies in the fact that it maps the exactly computable correlation functions of a supersymmetric parent theory into its non-supersymmetric daughter.

For the purpose of this short note, we take the parent theory to be $\mathcal{N}=1$ super Yang-Mills, with gauge group $U(2 N)$. We choose to orbifold by a $G=\mathbf{Z}_{2}$ action, which is embedded both within the gauge group and the non-anomalous $\mathbf{Z}_{4 N} \mathrm{R}$-symmetry. The resulting daughter is [6],

$$
U(N)_{1} \times U(N)_{2} \text { with a single Dirac fermion } \Psi=\left(\begin{array}{c}
\lambda \\
\bar{\psi}
\end{array}\right) \text { transforming as }(\mathbf{N}, \overline{\mathbf{N}}) .
$$

For later convenience, we have decomposed the Dirac fermion into its Weyl constituents, $\lambda$ and $\psi$. Each gauge group has the same coupling constant $g^{2}$ and, at one-loop, the dynamically generated scale $\Lambda^{3}=\mu_{0}^{3} e^{-8 \pi^{2} / g^{2} N}$ is chosen to coincide with that of the parent theory. The theta angles of the two groups are also set equal.

The diagonal $U(1)$ of the gauge group decouples, while the remaining $U(1)$ acts as a gauged baryon number current. We shall denote it as $U(1)_{B}$. The classical theory enjoys a further global, chiral $U(1)_{A}$ symmetry, acting as $\Psi \rightarrow \exp \left(i \gamma_{5} \alpha\right) \Psi$. The anomaly ensures that only a $\mathbf{Z}_{N}$ subgroup survives quantisation. Finally, the theory also retains memory of its orbifold birth through a $G=\mathbf{Z}_{2}$ global symmetry. Often referred to as the "quantum symmetry", it acts by exchanging the two gauge groups, while mapping $\lambda \leftrightarrow \psi$. Operators that carry charge under $G$ are known as "twisted operators" and descend from non-gauge-invariant operators in the parent theory.

For $N=3$, this theory is simply the chiral limit of QCD with three massless quarks, 
and the vector flavour group gauged. In analogy with QCD, it is expected that the chi$\mathrm{ral} \mathbf{Z}_{N}$ symmetry is spontaneously broken by a condensate of bi-fundamental fermions, resulting in $N$ vacuum states of the theory [5]. In the parent super Yang-Mills theory, the corresponding gluino condensate is exactly calculable and, assuming that the orbifold conjecture holds true, presents us with an exact prediction for the condensate in the orbifold theory [5, 7, 8 ]

$$
\langle\lambda \psi\rangle=32 \pi^{2} \Lambda^{3} e^{i(\theta+2 \pi k) / N}
$$

where $k=1, \ldots, N$ labels the $N$ degenerate vacua of the theory.

It was pointed out in [8] that, rather than discarding our holomorphic comfort blanket, we have instead wrapped a non-supersymmetric theory in it. This comment is motivated by the observation that the conjectured fermion condensate is holomorphic in the complexified coupling constant $\tau=\theta / 2 \pi+4 \pi i / g^{2}$. This dependence is fixed by the one-loop RG flow, together with the anomaly.

The original motivation of this paper was to test the prediction (11). A direct strong coupling instanton calculation in four dimensions gives a non-zero result only for $\left\langle(\lambda \psi)^{N}\right\rangle$. This is a familiar story from super Yang-Mills theories where a useful strategy to overcome the problem is to compactify the theory on a spatial circle of radius $R$, endowing the fermions with periodic boundary conditions. Introducing a Wilson line has the dual advantage of making the theory weakly coupled, and introducing the relevant fractional instanton configurations, allowing for a controlled semi-classical calculation of the condensate [9]. It is known that, as $R$ is varied, super Yang-Mills does not undergo a chiral phase transition and, in fact, the gluino condensate is independent of $R$. This is to be contrasted with thermal compactifications of this theory, in which the fermions have anti-periodic boundary conditions and a chiral phase transition does occur.

Since the perturbative orbifold conjecture is a property of planar graphs, one may expect it to continue to hold on $\mathbf{R}^{1,2} \times \mathbf{S}^{1}$. Moreover, the possibility of working at weak-coupling after compactification makes this a tempting path to follow, and was previously advocated in [7. Therefore, in this short note we shall consider the periodic compactification of the non-supersymmetric orbifold theory. In the next section we discuss perturbative results, turning to non-perturbative effects in the following section. We end with a short summary.

\section{The Effective Potential and Vacuum Physics}

One advantage of compactifying, say, the $x^{1}$ direction on a circle of radius $R$ is that one may introduce Wilson lines to break the gauge group to the maximal torus and 
A)

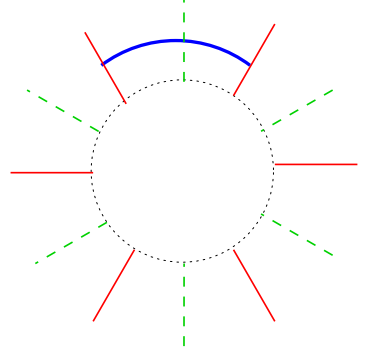

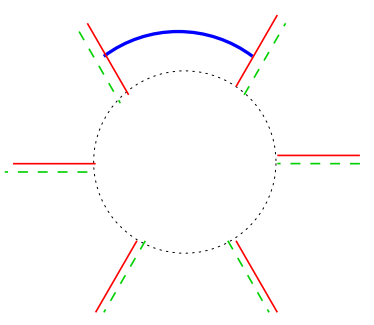

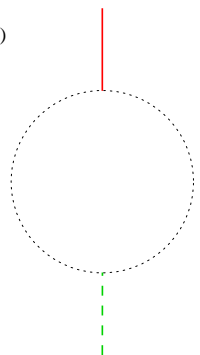

Figure 1: Three classical vacua of the theory. The solid (red) lines depict the $\mathbf{S}^{1}$ valued eigenvalues of the Wilson line for the $U(N)_{1}$ gauge group, while the dotted (green) lines depict the eigenvalues for $U(N)_{2}$. Calorons are shown as (blue) arcs connecting the eigenvalues of a given gauge group. This graphical representation finds life in the T-dual brane picture.

thus, for $R \ll 1 / \Lambda$, to a weakly coupled theory. We work with the Lie algebra valued object,

$$
\int_{0}^{2 \pi R} A_{1}^{i} d x^{1}=\operatorname{diag}\left(v_{1}^{i}, \ldots, v_{N}^{i}\right) \quad i=1,2
$$

Large gauge transformations imply the periodicity of the eigenvalues, and we may take each to lie in the range $v_{a}^{i} \in[0,2 \pi)$. The matter content of the theory is invariant under the diagonal $Z_{N}$ center of the two $S U(N)$ groups, allowing also for a twisted large gauge transformation. However, this is swamped by the overall $U(1)$ which we choose to keep. The classical moduli space of vacua is therefore given by $\left(T^{N} / S_{N}\right)^{2}$, where $S_{N}$ is the Weyl group of $U(N)$. We may choose to fix the Weyl symmetry by insisting $v_{a} \leq v_{a+1}$. It will prove useful to depict the different classical vacua graphically as two distinct sets of eigenvalues distributed around a circle as shown in Figure 1.

Firstly, let us recall what becomes of the vacuum moduli space in the supersymmetric parent theory. One finds that the classical vacuum moduli space survives at the perturbative level. However, fractional instantons, of the type considered in the following section, act as a repulsive force, pushing apart the eigenvalues of the Wilson line [10, 9]. In this manner, the theory is driven to weak coupling by compactification.

What does this supersymmetric vacuum descend to in the orbifold theory? In fact this point is already a little unclear. On graphical grounds, one may expect that the corresponding configuration consists of maximally separated, interlaced eigenvalues as depicted in Figure $1 \mathrm{~A}$

$$
v_{a}^{1}=v_{a}^{2}-\pi / N=2 \pi a / N \quad a=1, \ldots, N
$$

However, in this vacuum the twisted operator $\int A_{1}-A_{2}$ has a non-vanishing expectation value. To ensure that this operator vanishes, we should instead restrict to vacua of the 
daughter theory with $v_{a}^{1}=v_{a}^{2}$. If we insist that these eigenvalues are equally spaced, then we have the vacuum depicted in Figure 1B

$$
v_{a}^{1}=v_{a}^{2}=2 \pi a / N \quad a=1, \ldots, N
$$

However, as we shall now see, the discussion of which vacuum to pick is somewhat moot since the daughter theory will happily pick one for us. Given the matching of planar graphs 11, 2, 3, 4, one may think that the perturbative stability of any vacuum state is similar to super Yang-Mills, at least at large $N$. This can be checked by computing the one-loop contribution to the potential,

$$
\mathcal{F}=-\frac{1}{2 \pi R V} \log \left(\frac{\operatorname{det}^{2}\left(-D_{f}^{2}\right)}{\operatorname{det}\left(-D_{1}^{2}\right) \operatorname{det}\left(-D_{2}^{2}\right)}\right)
$$

where $V$ is the volume of the three-dimensional uncompactified space. The factors in the denominators come from integrating over the ghosts and gauge fields, with $D_{i}$ acting in the adjoint representation of the $U(N)_{i}$ gauge group. The numerator arises from integrating out the fermions, with $D_{f}$ in the bi-fundamental representation.

To compute the determinants, we borrow commonplace techniques from thermal field theory, remembering that our fermions are endowed with periodic boundary conditions. The relevant computation may be found in Appendix D of 11], where the basic function $\Delta=\log \operatorname{det}\left(-\left(\partial_{\mu}+(v / 2 \pi R) \delta_{\mu 4}\right)^{2}\right)$ is calculated on $\mathbf{R}^{3} \times \mathbf{S}^{1}$

$$
\begin{aligned}
\Delta & \equiv \frac{V}{(2 \pi)^{3}} \sum_{n=-\infty}^{\infty} \int d^{3} k \log \left(k^{2}+\frac{(2 \pi n-v)^{2}}{(2 \pi R)^{2}}\right) \\
& =\Delta_{0}+\frac{2 V}{(2 \pi)^{3}} \operatorname{Re} \int_{-\infty+i \epsilon}^{+\infty+i \epsilon} d k_{0} \int d^{3} k \frac{\log \left(k_{0}^{2}+\mathbf{k} \cdot \mathbf{k}\right)}{\exp \left(-i k_{0} / 2 \pi R-i v\right)-1} \\
& =\Delta_{0}+\frac{2 V}{(2 \pi)^{3}} \int d^{3} k \operatorname{Re} \log \left(1-e^{-k / 2 \pi R+i v}\right) \\
& =\Delta_{0}-\frac{2 V}{\pi^{2}} \frac{1}{(2 \pi R)^{3}} \sum_{j=1}^{\infty} \frac{\cos (v j)}{j^{4}}
\end{aligned}
$$

Here $\Delta_{0}$ is the divergent determinant evaluated on $\mathbf{R}^{4}$. It is independent of both $R$ and $v$, and is dealt with using a regularisation compatible with the supersymmetry of the parent theory, for example Pauli-Villars. If we take $v$ to lie in the range $v \in[0,2 \pi)$, then the final sum may be explicitly performed,

$$
\Delta-\Delta_{0}=-\frac{2 V}{4 \pi^{5} R^{3}}\left(\frac{\pi^{4}}{90}-\frac{v^{2}}{48}(v-2 \pi)^{2}\right) \quad \text { for } v \in[0,2 \pi)
$$


Using this result to calculate $\mathcal{F}$, we find that the $\Delta_{0}$ terms and the $\pi^{4} / 90$ terms cancel between bosons and fermions. This is a manifestation of the parent supersymmetry. A finite contributions to the potential remains,

$$
\begin{gathered}
\mathcal{F}=-\frac{1}{24 \pi^{2}(2 \pi R)^{4}} \sum_{a, b=1}^{N}\left[2\left[v_{a}^{1}-v_{b}^{2}\right]^{2}\left(\left[v_{a}^{1}-v_{b}^{2}\right]-2 \pi\right)^{2}-\left[v_{a}^{1}-v_{b}^{1}\right]^{2}\left(\left[v_{a}^{1}-v_{b}^{1}\right]-2 \pi\right)^{2}\right. \\
\left.-\left[v_{a}^{2}-v_{b}^{2}\right]^{2}\left(\left[v_{a}^{2}-v_{b}^{2}\right]-2 \pi\right)^{2}\right]
\end{gathered}
$$

where the square brackets are there to remind us that all periodically valued quantities live in the range $0<\left[v_{a}^{i}-v_{b}^{j}\right] \leq 2 \pi$. The physics behind this potential is clear: the first term arises from the fermions which contribute a negative mass to the Wilson line and push the eigenvalues apart; the next two terms come from the gluons which contribute a positive mass, attracting the eigenvalues. In the supersymmetric theory, these two contributions cancel and no potential is generated at one-loop. Here however the bosons and fermions carry different gauge quantum numbers leading to the form (66) for the potential. Similar potentials were found to lift flat directions in certain four-dimensional orbifold field theories with classical moduli spaces [12, 13]. In that case, the potential arose from UV divergent effects through a Coleman-Weinberg mechanism. The potential (6), like those of [12, 13], involves a double trace term for twisted operators, and is not suppressed at large $N$. A simple way to understand that, despite planar equivalence, the orbifold theory doesn't obey its supersymmetric parent is to observe that the boson and fermion mass matrices differ in a generic vacuum. In general, any orbifold theory with flat directions for twisted fields will suffer from a similar problem.

We may now judge the fate of the two vacua (3) and (4): both have vanishing vacuum energy, $\mathcal{F}=0$. Indeed, any vacuum in which the twisted operators vanish with $v_{1}^{a}=v_{2}^{a}$ has vanishing vacuum energy. Once again, this reflects the supersymmetric properties of the theories' ancestors. However, these supersymmetric configurations are not the vacua of the theory. This honour falls to the Wilson line depicted in Figure 1C,

$$
v_{a}^{1}=0 \text { and } v_{a}^{2}=\pi \quad \text { for } a=1, \ldots, N
$$

which boasts a vacuum energy of $\mathcal{F}=-N^{2} / 3.2^{6} \pi^{2} R^{4}$.

So what is the infra-red physics in the true vacuum? The full non-abelian gauge symmetry is restored, and the Wilson line (7) may be thought of as lying only in $U(1)_{B}$. This dynamically generated Wilson line gives the fermions a Kaluza-Klein mass $m=1 / 2 R$. To see the effect of this mass, consider the decomposition of the fermions in Fourier modes along the compact circle $\Psi\left(x_{1}, x\right)=\Psi_{n}(x) e^{i n x_{1} / R}$. Recall that our 
fermions are periodic and therefore, unlike a thermal Matsubara decomposition, the $n$ take values in the integers. In the background of the Wilson line (17), the kinetic term for the fermion is

$$
\Psi^{\dagger} \gamma_{u}\left(\partial_{\mu}-i\left(A_{\mu}^{1}-A_{\mu}^{2}\right)\right) \Psi=\sum_{n} \Psi_{-n}^{\dagger}\left(\gamma_{i} \partial_{i}-i \gamma_{1}\left(\frac{n}{R}+m\right)\right) \Psi_{n}
$$

We see that the effect of the mass term $m=1 / 2 R$ is to shift the moding of the fermions to half-integers. The gauge fields remain integer moded. Our theory has therefore driven itself to a thermal compactification. If we Wick rotate to Euclidean signature, all correlation functions will be those of a high temperature equilibrium thermal field theory. The resulting physics is well known. We expect that, at small $R \ll \Lambda^{-1}$, no condensate forms and the discrete chiral symmetry is restored. In analogy with QCD, it seems likely that a phase transition occurs at $R=R_{c}$. Needless to say, it would be interesting to understand the nature of the phase transition. In QCD with $N_{f}$ massless flavours, it is known that the chiral phase transition is second order for $N_{f}=2$, and first order for $N_{f} \geq 3$ [14]. However, since this analysis is based on the breaking of the continuous $S U\left(N_{f}\right)_{L} \times S U\left(N_{f}\right)_{R}$ symmetry, we should be cautious in extrapolating to our theory with only a discrete chiral symmetry.

\section{Fractional Instantons on $\mathbf{R}^{3} \times \mathbf{S}^{1}$ and the Condensate}

We have seen that, at least for $R \ll \Lambda$, the orbifold theory is dynamically driven to the vacuum (77) where the full non-abelian gauge symmetry is restored. In this vacuum, there are no semi-classical fractional instanton configurations and, indeed, we have argued above that the discrete chiral symmetry remains unbroken.

What happens above the critical radius, $R>R_{c}$ ? Perhaps the Wilson line (3) or (4) and the associated fractional instantons play an important role in the formation of the condensate? Interestingly, recent lattice simulations of $S U(2)$ gauge theory at finite temperature suggest that both the Wilson line and fractional instanton are indeed present at temperatures $T \leq T_{c}$ [15]. Motivated by this observation, in this section we examine the the fermionic zero mode structure of the available instanton solutions in the vacua (3) and (41), and calculate the corresponding condensate. Of course, this calculation is valid only in the regime $R \ll R_{c}$, and its value is correspondingly dubious.

The fractional instantons that appear in theories compactified on $\mathbf{R}^{2,1} \times \mathbf{S}^{1}$ are also known as "calorons". If the gauge group is broken to the maximal torus by a Wilson line, then each factor of $S U(N)_{i}$ plays host to $N$ "minimal calorons" [16] which carry only four bosonic zero modes (3 translation, and 1 gauge rotation). All other classical solutions are composed of these objects. Of these $N$ minimal calorons, $N-1$ are simply monopole solutions which are independent of $x^{1}$ and have action $4 \pi\left(v_{a+1}-v_{a}\right) / g^{2}$ for 
$a=1, \ldots, N-1$. These are accompanied by one further "Kaluza-Klein" monopole solution, related to the originals by an $x^{1}$-dependent gauge transformation. It has action $4 \pi\left(2 \pi-\left(v_{N}-v_{1}\right)\right) / g^{2}$. For the Wilson lines (3) and (41), we see that each of these minimal calorons, in each gauge group, has action $8 \pi^{2} / g^{2} N$.

The ability of a given instanton solution to contribute to $\langle\lambda \psi\rangle$ depends on the structure of its fermionic zero modes. In our case, the existence of these zero modes depends strongly on the choice of classical Wilson lines ${ }^{1}$. To see this, let us examine the Callias index theorem for monopoles in the presence of fundamental fermions. The important point is the dependence of the zero mode on the Kaluza-Klein mass of the fermions, which was studied in the Appendix of [17]. This Kaluza-Klein mass is a term of the form $i m \Psi^{\dagger} \gamma_{1} \Psi$ as seen in (8) which is not Lorentz invariant in $d=3+1$, but is permitted in $d=2+1$, where it is also known as a "real mass". The result of [17] is that each Weyl fermion in the fundamental representation of the gauge group donates a single zero mode to the $a^{\text {th }}$ caloron if its real mass $m$ lies within the range $v_{a}<m<v_{a+1}$.

Returning to the orbifold theory, consider a caloron in gauge group $U(N)_{1}$. It sees $N$ "flavours" of Weyl fermions transforming in the fundamental representation, with real masses determined by the Wilson line of $U(N)_{2}$. At this point, the utility of the pictorial representation of Figure 1 becomes apparent. A caloron solution in $U(N)_{1}$ is depicted by a arc stretching between two solid lines. Each dotted line corresponds to two Weyl fermions seen by this caloron. These Weyl fermions carry fermionic zero modes only if they intersect the caloron arc.

What happens for fractional instantons in the untwisted vacuum (44) of Figure 1B? As we can see from the picture, the dotted lines only barely touch the arc at the ends. From the gauge theory perspective, this corresponds to the limit in which the real mass coincides with the expectation value $m \rightarrow v_{a}$, at which point the number of fermionic zero modes jumps discontinuously. In fact, what happens is that the fermionic zero mode becomes non-normalizable at this point. One may suspect that this means that there is no caloron contribution to the fermion condensate in this vacuum. This suspicion is confirmed by an explicit computation of the condensate for arbitrary real mass, subsequently taking the limit as $m \rightarrow v^{a}$ (see the formulas in the second paper of [18]).

What about the Wilson line (3) depicted in Figure 1A? The eigenvalues of the $S U(N)_{1}$ and $S U(N)_{2}$ gauge groups are interlaced around the circle. Thus each minimal caloron solution receives zero modes from only a single flavour of Weyl fermion: each carries two fermionic zero modes, one for $\lambda$ and one for $\psi$. In short, all minimal

\footnotetext{
${ }^{1}$ At this point we differ from [7. Note that, as described previously, we allow for large gauge transformations which are twisted under the $\mathbf{Z}_{N}$ center, which also differs from [7]. I thank Misha Shifman for discussions on these issues.
} 
calorons are ideal candidates to give rise to the condensate (11). Summing over all minimal caloron contributions, following [9] closely, we find

$$
\langle\lambda \psi\rangle=2 N \int d^{3} X d \Omega \frac{J_{B}}{(2 \pi)^{4}} \int d^{2} \xi \frac{1}{J_{F}} \lambda^{(0)}(X) \psi^{(0)}(X)\left(\frac{\Gamma}{\Gamma_{0}}\right) e^{-8 \pi^{2} / g^{2} N+i \theta / N}
$$

The overall factor of $2 N$ reflects the number of minimal calorons in this model. Each has three position collective coordinates $X$ and a phase collective coordinate $\Omega$. The bosonic Jacobians for these were calculated in [18, 9] and give $J_{B}=2^{6} \pi^{4} R / N$. The two Grassmannian collective coordinates $\xi$ are saturated by the zero mode insertions of $\lambda^{(0)}$ and $\psi^{(0)}$. The Jacobian for fundamental fermions was calculated in [18, but in fact partially cancels the integration $\int d^{3} X d^{2} \xi$ over the zero modes, leaving behind a factor of $1 / 2 \pi R$. Finally, we come to the determinants, $\Gamma$. In the background of a self-dual field configuration they may be written as

$$
\Gamma=\left(\frac{\operatorname{det}^{1 / 2}\left(-\not D_{1} \not D_{1}^{\dagger}\right)}{\operatorname{det}^{\prime}\left(-\not D_{1}^{\dagger} \not D_{1}\right)}\right)\left(\frac{\operatorname{det}^{1 / 2}\left(-\not D_{2} \not D_{2}^{\dagger}\right)}{\operatorname{det}^{\prime}\left(-\not D_{2}^{\dagger} \not D_{2}\right)}\right) \operatorname{det}^{\prime 1 / 2}\left(\begin{array}{cc}
-\not D_{f} \not D_{f}^{\dagger} & 0 \\
0 & -\not D_{f}^{\dagger} \not D_{f}
\end{array}\right)
$$

The first two factors come from integrating over the ghosts (numerators) and gauge fields (denominators), with $D_{i}$ acting in the adjoint representation of the $U(N)_{i}$ gauge group. The final factor arises from integrating out the fermions, with $D_{f}$ in the bifundamental representation. All operators are evaluated on the background of the caloron and det' denotes the removal of zero modes. $\Gamma_{0}$ is the same operator evaluated on the vacuum, was found in the previous section to be $\Gamma_{0}=1$ in the background (3).

In the background of the caloron, $\Gamma$ is UV divergent. Introducing a cut-off mass scale $\mu_{0}$ through Pauli-Villars regularisation, the leading order contribution to the divergence arises from the truncation of the four bosonic and two fermionic zero modes. Since $4-\frac{1}{2} \times 2=3$, we have $\Gamma=c \mu_{0}^{3}$, with some real coefficient $c$. Further corrections are suppressed by $1 / R \mu_{0}$. Putting everything together, and taking the cut-off to infinity, we have

$$
\langle\lambda \psi\rangle=32 \pi^{2} c \mu_{0}^{3} e^{-8 \pi / g^{2} N} e^{i \theta / N}
$$

where $c$ is an $R$-independent constant which, for $c=1$, results in the claimed condensate (11). The above condensate is also holomorphic in $\tau$. In the semi-classical regime, such holomorphy reflects the fact that certain correlators are saturated by pure instanton contributions. For supersymmetric theories, this is assured by the extra goldstinos that arise for non-BPS configurations. In the present case, holomorphy holds only in the dilute gas approximation, where a background of $n_{+}$well-separated instantons and $n_{-}$anti-instantons carries $\left(n_{+}+n_{-}\right)$(approximate) fermionic zero modes. This is too many to contribute to $\langle\lambda \psi\rangle$. 


\section{Summary}

We have argued that super Yang-Mills and its orbifold exhibit qualitatively different behaviour when compactified on small circles of radius $R \ll \Lambda^{-1}$. Specifically, in super Yang-Mills the fermionic condensate is independent of $R$, while the orbifold theory undergoes a phase transition at some $R=R_{c}$, below which the discrete chiral symmetry is restored. This effect is due to the condensation of twisted operators, which take the orbifold theory away from the vacuum of its parent where the orbifold conjecture may be tested. Thus, the disappointing conclusion of this note is that compactification on a spatial circle is not a useful way to test the orbifold conjecture.

A glimmer of hope remains however. We have shown that in the presence of the Wilson line (3), the fractional instanton has the correct zero mode structure to give rise to the conjectured fermion condensate. Hints from the lattice [15] suggest that this Wilson line and the associated fractional instanton may play a role at $R>R_{c}$.

It is perhaps worth making one final comment. For super Yang-Mills, the condensate is independent of $R$ for all $N$. Is there similar behaviour for the orbifold theory for $R>R_{c}$ at large $N$ ? Once again, we may look to the analogy with QCD, now at low temperatures. As the temperature is increased from zero, the chiral condensate is known to decrease as $T^{2} / f_{\pi}^{2}$ [19. Since $f_{\pi} \sim \mathcal{O}\left(N^{1 / 2}\right)$, this suggests that, at low temperatures, the condensate does indeed remain constant in the large $N$ limit.

\section{Acknowledgments}

I'd like to thank Luis Bettencourt, Jiunn-Wei Chen, John Negele, York Schroder, D.T. Son, Matt Strassler, Jan Troost and especially Ami Hanany and Andy Neitzke for many useful discussions. I'd also like to thank Allan Adams and Misha Shifman for extensive comments and discussions on the draft. I'm supported by a Pappalardo fellowship, and am very grateful to Neil Pappalardo for the money. This work was also supported in part by funds provided by the U.S. Department of Energy (D.O.E.) under cooperative research agreement \#DF-FC02-94ER40818.

\section{References}

[1] S, Kachru and E. Silverstein, "4d Conformal Field Theories and Strings on Orbifolds", Phys.Rev.Lett. 80 (1998) 4855, hep-th/9802183.

[2] A. Lawrence, N. Nekrasov and C. Vafa, "On Conformal Theories in Four Dimensions", Nucl.Phys. B533 (1998) 199, hep-th/9803015.

[3] M. Bershadsky, Z. Kakushadze and C. Vafa, "String Expansion as Large $N$ Expansion of Gauge Theories", Nucl.Phys. B523 (1998) 59, hep-th/9803076. 
[4] M. Bershadsky and A. Johansen, "Large N limit of Orbifold Field Theories", Nucl.Phys. B536 (1998) 141, hep-th/9803249.

[5] M. Strassler, "On Methods for Extracting Exact Non-Perturbative Results in NonSupersymmetric Gauge Theories", hep-th/0104032

[6] M. Schmaltz, "Duality of Non-Supersymmetric Large N Gauge Theories", Phys. Rev. D59 (1999) 105018, hep-th/9805218.

[7] A. Gorsky and M. Shifman, "Testing Nonperturbative Orbifold Conjectures", hep-th/0208073

[8] R. Dijkgraaf, A. Neitzke and C. Vafa, "Large $N$ Strong Coupling Dynamics in NonSupersymmetric Orbifold Field Theories", hep-th/0211194

[9] M. Davies, T. Hollowood, M. Mattis and V. Khoze, "Gluino Condensate and Magnetic Monopoles in Supersymmetric Gluodynamics", Nucl.Phys. B559 (1999) 123, hep-th/9905015

[10] S. Katz and C. Vafa, "Geometric Engineering of $N=1$ Quantum Field Theories", Nucl. Phys. B497, 196 (1997), hep-th/9611090

[11] D. Gross, R. Pisarski and L. Yaffe, "QCD and Instantons at Finite Temperature" Rev. Mod. Phys. 53, 43 (1981).

[12] A. Tseytlin and K. Zarembo, "Effective Potential in Non-Supersymmetric $S U(N) \times$ $S U(N)$ Gauge Theory and Interactions of Type 0 D3-Branes", Phys. Lett. B457, 77 (1999), hep-th/9902095.

[13] A. Adams and E. Silverstein, "Closed String Tachyons, AdS/CFT, and Large N QCD", Phys.Rev. D64 (2001) 086001, hep-th/0103220.

[14] R. Pisarski and F. Wilczek, "Remarks on the Chiral Phase Transition in Chromodynamics", Phys. Rev. D29, 338 (1984).

[15] E. Ilgenfritz, B. Martemyanov, M. Muller-Preussker, S. Shcheredin and A. Veselov, "On the Topological Content of SU(2) Gauge Fields Below T(c)", Phys. Rev. D66, 074503 (2002), hep-lat/0206004.

[16] K. Lee and P. Yi "Monopoles and Instantons on Partially Compactified D-Branes", Phys. Rev. D56 3711 (1997), hep-th/9702107.

T. Kraan and P. van Baal, "Periodic Instantons with Non-Trivial Holonomy", Nucl. Phys. B533, 627 (1998), hep-th/9805168

[17] J. de Boer, K. Hori and Y. Oz, "Dynamics of $N=2$ Supersymmetric Gauge Theories in Three Dimensions", Nucl. Phys. B500, 163 (1997), hep-th/9703100.

[18] N. Dorey, V. Khoze, M. Mattis, D. Tong and S. Vandoren, "Instantons, ThreeDimensional Gauge Theory, and the Atiyah-Hitchin Manifold", Nucl. Phys. B502, 59 (1997), hep-th/9703228.

N. Dorey, D. Tong and S. Vandoren, "Instanton Effects in Three-Dimensional Supersymmetric Gauge Theories with Matter", JHEP 9804, 005 (1998), hep-th/9803065.

[19] J. Gasser and H. Leutwyler, "Thermodynamics Of Chiral Symmetry", Phys. Lett. B188, 477 (1987). 